\title{
OPTIMIZATION MODELS FOR EMERGENCY RESPONSE AND POST- DISASTER DELIVERY LOGISTICS: A REVIEW OF CURRENT APPROACHES
}

\author{
Sampson E. Akwafuo ${ }^{*}{ }^{\mathbb{}}$, Armin R. Mikler ${ }^{1}$, Fariba A. Irany ${ }^{1}$ \\ ${ }^{*}$ Center for Computational Epidemiology and Response Analysis (CeCERA), Department of \\ Computer Science and Engineering, University of North Texas, Denton, USA
}

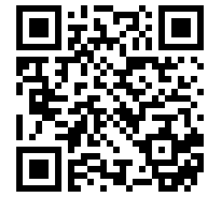

DOI: https://doi.org/10.29121/ijetmr.v7.i8.2020.738

Article Citation: Sampson E.

Akwafuo, Armin R. Mikler, and

Fariba A. Irany. (2020).

OPTIMIZATION MODELS FOR

EMERGENCY RESPONSE AND POST-

DISASTER DELIVERY LOGISTICS: A

REVIEW OF CURRENT

APPROACHES. International Journal of Engineering Technologies and Management Research, 7(8), 35-49. https://doi.org/10.29121/ijetmr.v7 i8.2020.738

Published Date: 27 August 2020

Keywords:

Disasters

Public Health Emergencies

Vehicle Routing Problem Algorithms Heuristics

\begin{abstract}
Emergency response preparedness increases disaster resilience and mitigates its possible impacts, mostly in public health emergencies. Prompt activation of these response plans and rapid optimization of delivery models and are essential for effective management of emergencies and disaster. In this paper, existing computational models and algorithms for routing deliveries and logistics during public health emergencies are identified. An overview of recent developments of optimization models and contributions, with emphasis on their applications in situations of uncertainties and unreliability, as obtainable in low-resource countries, is presented. Specific recent improvements in biologically-inspired and intelligent algorithms, genetic algorithms, and artificial immune systems techniques are surveyed. The research gaps are identified, and suggestions for potential future research concentrations are proffered.
\end{abstract}

\section{INTRODUCTION}

Emergencies and disasters have continued to plague humans for ages. In recent years, however, there has been a tremendous increase in their frequency of occurrence, and this may be attributed to the increase in reportage of these events. A disaster occurs when there is an ecological breakdown of relationships between man and his environment (Al-dahash, Thayaparan, and Kulatunga 2016). It is considered a serious disruption to man's usual activities. It may be of natural causes, including flooding, hurricanes, earthquakes or man-made, such as civil strife, terrorism, or bio-weapon attack. Within the last decade, 3684 natural disasters have occurred globally, resulting in 684,279 deaths (Ritchie, H; Roser 2019). These disasters, in addition to man-made situations and public health emergencies, often result in massive demands that exceed the local capacity to respond or outstrip available resources. Existing infrastructure and facilities crumble, thereby making response planning difficult if there is no existing plan. Immediate activation of an existing plan, with few modifications where necessary, is needed to effectively mitigate damages and losses. The focus of this review is on public health emergency situations, such as disease outbreaks and evacuations.

In public health, impacts of emergencies and disasters are broad, highly diverse and remarkably devastating. These situations include sudden infectious disease outbreaks, loss of safe water or food supply and health

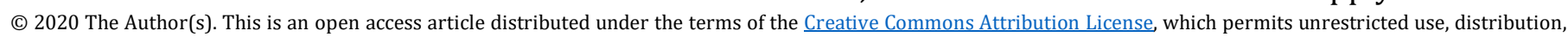
and reproduction in any medium, provided the original author and source are credited. 
consequences of large-scale societal conflicts. Public health officials play intrinsic roles in the disaster preparatory and delivery system. At the national level, they ensure mitigation of disaster impacts by preparing the response plan, for subsequent optimization. At the community level, they build disaster resilience by assessing the peculiar needs of the locality, in the event of disaster or epidemics outbreak and ensuring prompt implementation of the plan during the post-disaster period (Keim 2008). Operations of an emergency response plan, from the national relief products reservoir to the end-users, as obtainable in most Low and Middle-Income Countries (LMICs) are depicted in Fig. 1. An effective concerted emergency planning and response delivery activities involve at least six activities as described in (Center for Disease Control and Prevention (CDC): Emergency Preparedness Report 2016): These include (1) Community resilience: This studies the ability of the locality to withstand, adapt and recover from a disaster or public health emergency. (2) Countermeasures and Mitigation, involving all pro-active measures to ensure appropriate medications, vaccinations and interventions are ready; and delivering them as at when due, to alleviate impacts of these emergencies. (3) Surge Management, which involves the ability to re-route or re-allocate resources, to manage rapidly expanding large emergencies. (4) Information Management: this ensures the provision of accurate and timely information to the population about public health emergencies and expectations. (5) Biosurveillance, including the use of all available methods to survey, investigate and identify health threats, and lastly (6) Incidence management, which deals with activating and coordinating an effective response.

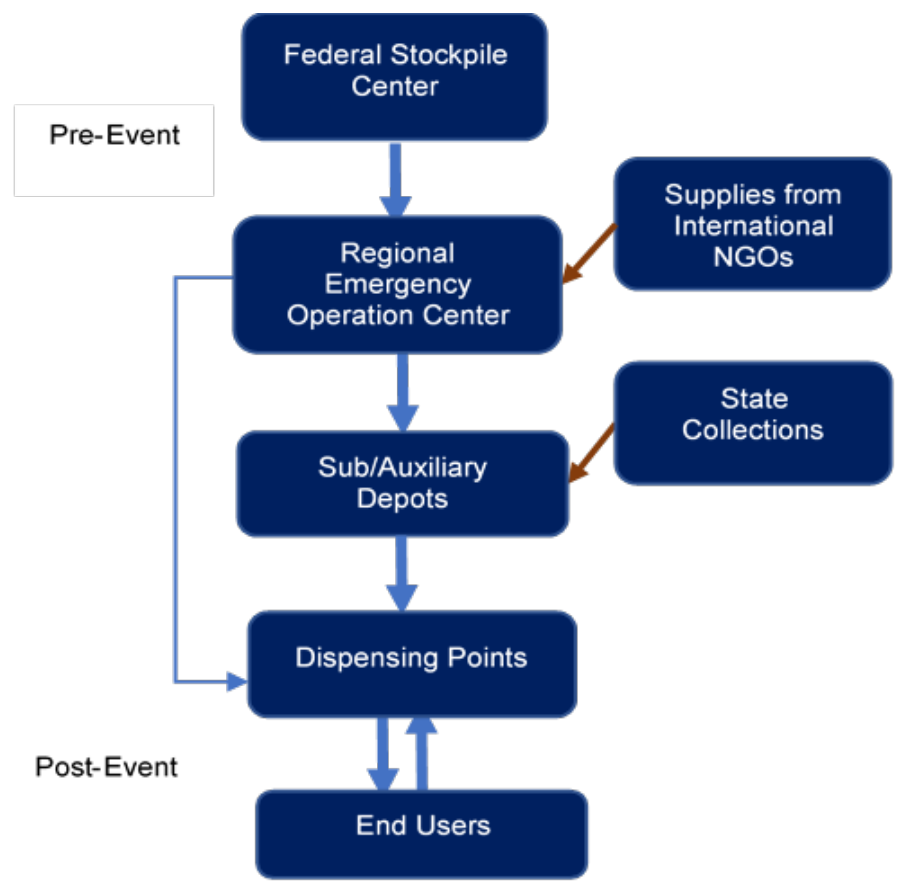

Figure 1: Operations of an emergency response plan in low-resource settings

Prompt activation of these response plans is imperative in reducing casualties during these situations. The activation ensures that medical countermeasures (MCMs) and other products are distributed to every person in need, directly to their locations, as quickly as possible. This reduces the severity of possible impacts of these events. In most low-resourced countries, the situation is immediately assessed by the responsible organization, and mobilization of resources and relief materials from Non-Governmental Organizations (NGOs) and foreign donors is embarked upon. This is usually coordinated by government agencies, such as public health departments (ogunwolu, L; Sosimi 2018). These activities include setting up emergency facilities/displaced person camps or Points of Dispensing (PODs), providing medical counter-measures and aids, scheduling transportation to the camps, and dispatching vehicles for relief supplies(Zheng, Chen, and Ling 2015). Logistics planning and routing of emergency management and deliveries, therefore, represent a very challenging task for public health response planners. It is a variant of Vehicle Routing Problem (VRP) and constitutes a combinatorial optimization problem. This and other variants of VRP has been found to be NP-hard. Therefore, polynomial-time algorithms to solve this problem are unlikely to exist unless $P=$ NP (Kumar and Panneerselvam 2012). Unlike other variants of VRP, emergency response 
planning and delivery present an arduous task, due to the following additional problems (Caunhye, Nie, and Pokharel 2012):

1) Unreliability and additional uncertainties: In a rapidly-changing situation, road networks, communication links and hitherto-available routes, may suddenly become unavailable. These infrastructures may not be wholly relied on during planning. There are also uncertainties in demand of affected locations.

2) Additional complex coordination issues: The involvement of many third parties, such as international non-governmental organizations, donor partners and local donations from some individuals or groups presents additional coordination issues. Accessibility to accurate real-time demand information becomes difficult in such situations.

3) Unpredictability in demand's changes: Hitherto available limited resources are often overwhelmed by spikes in demand. Existing emergency response plan may not cover these changes, due to their inherent unpredictability, coupled with supply changes and transportation capacity issues.

4) Prompt Delivery: There are some usual additional difficulties in achieving efficient and timely delivery during emergencies, due to changes in the transportation network.

A mathematical formulation of the general variant of the vehicle routing and logistics problem is presented in the next subsection. Optimization algorithms for Depot location-allocation are presented in section two. Section three discusses algorithms for optimizing route construction and route improvement, including evolutionary, artificial intelligence-based algorithms, and artificial immune systems. Other metaheuristic algorithms are presented in section four. Section five presents potential directions for future research and conclusion. The algorithms are reviewed under the following grouping: (1) Facility Location-Allocation Problem, (2) Routing Problem, and (3) Integrated approaches

\subsection{MATHEMATICAL FORMULATION OF THE GENERAL ROUTING PROBLEM}

The Vehicular Routing Problem essentially seeks to compute the optimal order of delivery routes, a fleet of vehicles must visit. It is denoted by an ordered list of locations or nodes in a graph. The prime objective is to find optimal routes for fleets of vehicles when visiting a set of sites for deliveries. Each location may have a unique demand representing the number or size of commodities required, or a time window in which the vehicle should arrive. It was initially introduced by Dantzig and Ramser (Dantzig and Ramser 1959) and different variations of VRP have emerged and generated a lot of interest over the years. The emergency vehicle routing problem is one of these variations and presents a challenging and complex task for public health officials.

VRP can, therefore, be represented by a graph $G=(V, E)$ where $V$ represents a set of vertex, $\mathrm{V}=\{0,1, \ldots n\}$ and $E$ is the set of edges $E=\{[i, j]: i, j \varepsilon V\}$. The central depot, Emergency Operating Center (EOC), or receiving, staging and storing (RSS) center is the location where all vehicle routes must start, and can be denoted by the index $0 \epsilon V$ and each $i$ in $V$ is a location or sub-depot that needs to be visited to deliver relief supply. This implies that $G$ is constructed as a complete graph. Therefore, any of these locations can be reached directly from the depot or through adjacent locations for a cost. This cost represented as a matrix, $C_{i j}$ is defined as part of the set $E$, and corresponds to the cost of traveling between locations $i$ and $j$. When $i=0, C_{i j}$ represents the cost between the depot and location $\mathrm{j}$. Even though it may not be realistic in all settings, it is assumed that this cost is always symmetric, $C_{i j}=C_{j i}$, and satisfy the triangle inequality: $C_{i j} \leq C_{i k}+C_{k j}, \quad \forall i, j, k \varepsilon V$. Usually, the upper bound of $C_{i j}$ is denoted by $z$, and is the maximum acceptable total travel time per route. Let $x_{i j}$ be the decision variable, where $x_{i j}=1$, if there is a vehicle, $\mathrm{v}$, which serves location $j$, from depot $i$; and $x_{i j}=0$ otherwise. The primary objective of the general VRP, according to (Urbanovsky 2018; Gai et al. 2015), is to find a solution, which will minimize the total cost and number of routes required to serve all locations within the allowed time. This is formulated below:

$$
\min \sum_{i \in V} \sum_{j \in V} C_{i j} x_{i j}
$$

Subject to: 


$$
\begin{aligned}
& \sum_{i, j \in V} x_{i j}^{v} \leq 1 \quad \forall i, j, \\
& \sum_{i, j, k \epsilon V} \pi x_{i j k} \leq z \\
& \sum_{i \in V}^{n} x_{i 0}=\sum_{j \in V} x_{0 j}=k \\
& \sum_{i \in V}^{n} d_{i} \leq \tau r_{i} \\
& \sum_{i \in S}^{n} x_{i j}^{v} \subseteq r(S), \forall S \subseteq V\{0\}, S \neq 0, v \varepsilon \\
& x_{i j} \epsilon\{0,1\} \quad \forall i, j \epsilon V
\end{aligned}
$$

The prime minimization objective is as shown in equation (1). Constraints (2) ensures that exactly one route enters and leaves each vertex associated with a node. The total travel time for any route is less or equal to the maximum allowed travel time, z. This is represented by constraint (3). For the general variant, constraint (4) indicates that the number of vehicles leaving the depot is the same as the number entering the same depot. However, our focus is on disaster and emergency management, this variant does not include the optimization of routes back to the depot. Constraint (5) is the capacity constraint. It ensures that the total demand on each route does not exceed the vehicle's capacity, assigned to that route. Constraint (6) ensures that there is a road or connection between different routes. $S$ is a route and part of the entire multi-depot network. Constraint (7) is the integrality constraints, ensuring that the decision metric lies between zero and one.

\section{OPTIMIZATION MODELS FOR EMERGENCY FACILITY LOCATION}

Accurately placing emergency facilities, such as depots, displaced persons' camps, shelters, etc to ensure optimal usage during public health emergencies is an intrinsic part of emergency management and logistics problem. Many factors are taken into consideration when planning the location of these facilities. These include the topography of the location, accessibility to different types and capacities of vehicles, road network to the location and proximity to potential end-users. There are usually four approaches to the problem, depending on the planners' perception of the problem: deterministic, dynamic, stochastic, and robust facility location problems. Establishing a single facility, to cover a maximum number of individuals or demand points is a simple form of facility location-allocation problem, introduced by studies in (Church, R. Velle 1974). However, finding the best locations for multiple sites is an NP-hard problem, as shown by studies in (Boonmee, Arimura, and Asada 2017), with the addition of smaller backup locations. For a deterministic location problem, different models are used. One of these is the minimax model, aimed at minimizing the transport cost for potential locations to end-users, and maximizing $D_{m}$; the possible number of facilities or depots.

Let $i$ a be a demand node belonging to a set of nodes $I$ and ( $i \epsilon I$ ) and $j$ represents a candidate facility site $(j \in J)$. For decision variables, $x_{j}=1$ if a facility is located at an eligible site $j$, and 0 otherwise. $\boldsymbol{y}_{\boldsymbol{i} j}=1$ if a facility $j$ services demand point $i$ and 0 otherwise. $\boldsymbol{d}_{\boldsymbol{i j}}$ represents the distance between the POD $i$ and the candidate facility location $j$. This Euclidean distance is calculated using Haversine formula and presented as a distance matrix (Anisya and Swara 2017). $w_{i}$ refers to the total demand of POD $i . j_{\max }$ is the total capacity of facility $j$ while $D_{m}$ is the maximum number of facilities that can be acceptably placed within the region of interest. The facility allocation problem can therefore be mathematically formulated as:

$$
\min \operatorname{loc}=\sum_{i} \sum_{j} w_{i} y_{i j} d_{i j}
$$

Subject to

$$
\begin{gathered}
\sum_{i} x_{j} \leq D_{m} \\
\sum_{j} Y_{i j}=1 \forall i \epsilon I
\end{gathered}
$$


Sampson E. Akwafuo, Armin R. Mikler, and Fariba A. Irany

$$
\begin{gathered}
\sum_{i} w_{i} Y_{i j} \leq j_{\max } X_{i} 1 \forall j \in J \\
x_{j}, Y_{i j} \in\{0,1\} \forall i, j
\end{gathered}
$$

The objective function (8) minimizes the total distance between POD locations and candidate facilities. According to Constraint (9), the total number of facilities located at site $j$ will be, at most, equal to $\boldsymbol{D}_{\boldsymbol{m}}$ the maximum number of possible facilities. Constraint (10) ensure that each POD $i$ is assigned to a facility, j, while constraint (11) allows assignment only to sites where an acceptable facility is located, and ensure that capacity of the local facility is not exceeded. The binary conditions for the model variables are maintained by constraint (12).

An exact method for solving a single-source, but two-tiered facility location problem was suggested in (Tragantalerngsak, Holt, and Ronnqvist 2001). Two echelons of facilities exist and facilities in the second echelon have limited capacities, and can be supplied by only one facility in the first echelon. A branch and bound algorithm based on Lagrangian relaxation is proposed. A similar but updated hybrid model for competitively locating new facilities and determining roles of each of the facilities is later proposed in (Bagherinejad and Niknam 2018). The authors proposed an algorithm for adjusting locations of facilities, which may involve closing down some facilities, while opening new locations, in accordance with newer information. The model is solved using a combination of Tabu Search and exact methods. The performance is compared with Karush Kuhn-Tucker upper bound conditions. Several factors are considered when planning real-world emergency facility location, mostly in large-scale operations. These include service priorities, redundant facilities and the dynamics of candidate locations, according to studies in (Boloori Arabani and Farahani 2013). Here, the authors suggested the consideration of trade-off between making location changes and cost of relief transportation to demand points. Some models are only concerned with allocation of facilities to ensure maximum coverage, while few of the suggested algorithms include relief distribution routing. These include approaches suggested by studies in(M. Shen, Dessouky, and Ordóñez 2007), (Alenezy 2020) and (Balcik and Beamon 2008). Maximal covering location models with are formulated and suggested in (Walia et al. 2018; Y. Han, Guan, and Shi 2011; L. Shen et al. 2019) includes some routing models for relief distribution. Some facility location models combine relief distribution and stock-prepositioning, aimed at achieving cost minimization. Studies in (Rawls and Turnquist 2010) and (Mete 2010) include some stochastic procedures for considering uncertainties in demand, stocked supplies, supplies as well as uncertainty regarding transportation network availability.

\subsection{GENETIC ALGORITHMS FOR FACILITY LOCATION}

Genetic algorithms (GA) are approximation approaches for solving combinatorial problems. They are based on Charles Darwin's principle of natural selection and the survival of the fittest in optimization. A GA works through series of genetic operations including crossover and mutation, in a pre-selected population, and selects the most appropriate offspring to pass on to next generations. To produce these offsprings, GA uses the natural selection process where the fittest individuals are selected for reproduction. It involves other procedures, such as a representation of chromosomes, the creation of initial population, reproduction and local search improvement. Binary strings are most often used to represent chromosomes in the GA technique. An integer string of length $N$ may be used to represent the chromosome. Each gene in the chromosome represents the node in a facility allocation problem and the sequence of the genes in the order of visiting these locations.

Some GA heuristics models suggest the consideration of the probability of catastrophic occurrence for a region, when planning locations of rescue center. Studies in (Wang, D., Zhang 2006) propose a mathematical model with an embedded genetic algorithm for consideration of probability of disaster occurrence, using data from disparate sources. A mathematical model with an embedded genetic algorithm was proposed. The use of a capacitated GA and p-median approach in solving the general variant of facility location problem is suggested in (Mangla, Akhare, and Sanjay 2015). The p-median problem is a specific type of a discrete location model, where $p$ facilities to located such that demand-weighted average distance between a demand point and the selected facility is minimized. A study of extended emergency facility location problem is presented in(C. F. Han and Zhang 2009) . The problem is modeled as an IP situation, and a GA is proposed where each chromosome is represented by the serial number of each target's emergency facility. The population crossover is obtained by using a changeable mutation probability. They integrated a GIS-based intelligent search method with a GA, aimed at obtaining an optimum spatial approach is 
Optimization Models for Emergency Response and Post-Disaster Delivery Logistics: A Review of Current Approaches

suggested (X. Li and Yeh 2006). Barzinpour et al (Barzinpour et al. 2014) proposed a similar model for bi-objective optimization of location-allocation in disaster relief planning and logistics. It aims to minimize the total costs of distribution, in terms of distance and transportation logistics, while maximizing the least rate of the satisfaction in distribution network. From these and other related publications, it can be deduced that GA are in the majority of facility location methodologies for emergency management. There is, however, opportunities for piloting new metaheuristics approaches, mostly hybridization of the GAs with other methods, such as machine learning and artificial intelligence-based approaches to possibly enhance performance.

\section{ROUTE CONSTRUCTION AND IMPROVEMENT MODELS}

Route construction is an intrinsic part of emergency management and logistics delivery. It involves finding the optimal order to visitation to all nodes in a selected cluster. It exploits all nodes in a given central facility location and independently constructs route through members of that cluster. Routes are checked to ensure compliance with demand constraints and vehicle capacities. Although some studies combined the two processes, most approaches adopt a two-step heuristic mechanism, where route construction precedes route improvement (Campbell, Vandenbussche, and Hermann 2010; Pichpibul and Kawtummachai 2013; Chen, Hsueh, and Chang 2006). The route construction phase generates initial feasible routes. These are inputs into the route improvement phase. In this phase, a heuristic permutation of selected $b$ routes is considered and $k$ locations are interchanged in the cross-mix mechanism. It evaluates all possible string lengths between a pair of routes, and increase $k$ when an evaluation cycle has been completed without identifying an improvement move. Hence, at each iteration, the results are evaluated until no more improvements or the seed is reached. It evaluates all the possible moves and implements the best one.

Dynamic vehicle routing problem (DVRP) has been suggested as a viable alternative for managing public health emergencies and natural disasters in LMICs. It is a class of VRP that handles requests from locations in real- time. It combines the two steps of traditional VRP (Planning and Execution) into a dual, same time process(Pillac et al. 2013). The concept of immediate request, which includes a call or demand from an affected location and requesting to be serviced as early as possible was first introduced by Psaraftis in (Psaraftis 1980). This will, therefore, induce an immediate re-planning of current vehicle route. The most common source of dynamic request in emergency vehicle routing management is the online arrival of customer requests when vehicles are already in operation. The online request system is continually monitored for arrivals, at the control station. These requests are usually demand for goods, an evacuation call or request for public health services as shown in (Hvattum, Løkketangen, and Laporte 2007; Ben-Akiva et al. 2012; Sever et al. 2018). Chen et al (Chen, Hsueh, and Chang 2006) proposed the use of critical nodes in a real-time, but time-defendant VRP, which was formulated as series of mixed-integer programming. Critical nodes are used to delineate the scope of remaining locations in a time rolling horizon. They also serve as points of dispensing (PODs) and service time at these nodes vary depending on the most current information received at the time of arrival of the vehicle. Vehicles routes, duration or travel time, and departure times were treated as decision variables. A technique for choosing optimal departure time and route selection heuristics was developed. To efficiently handle uncertainties in real-time, during emergencies, (Radianti et al. 2014) proposes a Dynamic Bayesian Network (DBN) to capture and process hazard and crowd dynamics. The model is based on a spatiotemporal probability. It is made up of processes for accessing the hazard of the location, the risk model of the possible routes and a flow model. Each model is subject to Markov conditions and used to predict the behavior and movement of individuals at the affected locations. A Markov condition assumes that every node in a Bayesian network is conditionally independent of its non-descendent or directly connected nodes. By using the hazard mode, the relationship between the POD location and the hazard status of the road network leading to the location are tracked and predicted. A hazard model computes the probability of the probability that the locality experience a disaster within a particular time-period. Hence, the probabilistic risk level of each location can be tracked from the model. This information is used in effectively updating the routing network, thereby minimizing the overall fatality network. Where there is a substantial absence of information about demand points and affected locations, a periodic dynamic methodology may be used. Such a periodic re-optimization process is highlighted in (AbdAllah, A.M; Essam, D. L; Sarker 2017), and provides an effective approach when number of nodes grows rapidly. The study by Che-fu et al (Hsueh, Chen, and Chou 2008) improve on the use of critical nodes and suggest demands consideration at each critical node. Any effective method for routing relief logistics following disasters, should include alternative depot locations, assuming these depots or ad-hoc storage centers become unavailable. Since demands and even supplies 
Sampson E. Akwafuo, Armin R. Mikler, and Fariba A. Irany

vary in real time in such situations, vehicles may route to a critical node in the first instance, while servicing all locations along the route.

\subsection{EVOLUTIONARY APPROACHES}

Some Genetic Algorithm-based approaches have been developed for effective management of emergency logistics. A hierarchical GA approach in which local optimum is achieved by a bottom algorithm in a subnet, while a global optimization is attained by using a top GA technique in the whole network is suggested in (Ma, Ye, and Hou 2011). It strives to maintain a balance between a GA random search for global search capability and a search efficiency using a narrow search technique. A rather complex approach for solving multi-items, such as different depots, during emergencies is proposed in (Lin et al. 2012). It uses multi-objective integer programming model to formulate a split delivery strategy. Soft time constraints and multi-period routing were considered in the new model's implementation. Studies in (Jotshi, Gong, and Batta 2009) proposed the use of data fusion, which greatly enhances information collation and analysis in a disaster scenario. This enables Emergency Response Centers to interpret and use massive amounts of data, including reports of current status of the locations, casualties, structural damage, road and traffic conditions.

\subsection{OTHER BIO-INSPIRED AND INTELLIGENT ALGORITHMS}

Artificial intelligent-based algorithms and methodologies have continually demonstrated capabilities for solving many real-word problems. They integrate many learning techniques and adapt to fast-changing situations, thereby helping to overcome some computational constraints and achieve faster results. These approaches have been proven to be very effective in obtaining optimal results for many variants of the vehicle routing problem(Simić and Simić 2012). Biologically inspired algorithms are motivated by biological mechanisms and are based on living beings' behaviors or observed natural phenomen. They include ant colony optimization, genetic algorithm, particle swarm optimization, evolutionary algorithms, neural networks and artificial immune system-inspired algorithms(Prasanth and Kandikonda 2015). However, few proposals for their applications in emergency settings have been suggested. Some of these well-known approaches and recent research publications on these techniques are highlighted below.

\subsubsection{ANT COLONY OPTIMIZATION}

Many approaches to emergency rescue operations and other response activities are based on some forms of ant colony optimization (ACO). It models the behavior of ants and other social insects and aids in the development of algorithms to solve difficult mathematical problems. It provides an advanced degree of reliability for obtaining shortest distance routing in EMVRP. ACO was initially proposed as an ant system by Dorigo at al (Bonabeau, E; Dorigo, M; Theraulaz 2000), and several variations have been suggested. These include changing rules of random search, to increase available space in the original ACO (Yu, B; Yang, Z; Yao 2009), introducing $N$-opt ( $N \geq 2$ ) level of searches, and its applications in a dynamic environment, such as an immigrant system modeling (Yang, S; Tinos 2007), and clustering of locations near selected phenomenon points(Gao, S; Wang, Y; Cheng, J; Inazumi, Y; Tang 2017). Ziyao li(Z. Li 2010) proposes a methodology for ranking of pheromone, based on elite ant visibility, thereby increasing the speed of convergence. It employs pheromone trail updating, where specified equations and local rules are used to intermittently adjust the route. An enhanced ant colony optimization, with an application of K-means for grouping regions of interest, is proposed in (Xu 2018). It uses crossover mechanics to improve solutions, in dynamic situations and could be used for emergency response planning.

The last mile distribution problem, which deals with serving population in need, through timely provision of relief materials from existing distribution centers, using a fixed set of homogenous vehicles is studied in (Ferrer, Ortuño, and Tirado 2020). There are provisions for seamless vehicle replacement, as there are chances that aid may be attacked while traveling or some parts of the road may become inaccessible due to the disaster. An ant colony optimization-based methodology is proposed, with two types of ants symbolizing vehicles and humanitarian aid kits and four types of pheromones, which is an indication of previous ants. The methodology aims to minimize six different attributes of Time, Cost, Equity, Priority, Security, Reliability. A methodology for providing nearby supplies 
Optimization Models for Emergency Response and Post-Disaster Delivery Logistics: A Review of Current Approaches

to anticipated new locations, such that eventual requests from these locations can be effectively serviced in real-time is proposed in (Xiang et al. 2020). This approach is termed ant colony optimization-based demand coverage diversity method (ACO-CD) and helps to maintain the multifariousness of location's requests at the next time slice by ensuring there is always a location close to the newly emerged location, which will result in low traveling cost. The demand coverage diversity adaptation method ensures that the optimizer can efficiently respond to the newly appeared customer requests by maintaining the heterogeneity of the covered locations in the route.

Path selection is one of the fundamental problems in emergency logistics management. Y. Yuan and D. Wang (Yuan and Wang 2009) propose two mathematical models for solving this problem and selecting the best path in large-scale emergencies. The first model aims to minimize travel time in a route, taking into account that the travel speed on each arc may be affected by different levels of disaster effect. The travel speed on each arc is modeled as a continuous decrease function with respect to time. A modified Dijkstra algorithm was proposed to solve the model. The chaos, panic, and congestions in times of disaster were also considered on the first model. The second model has multiple objectives: to reduce the time, and to minimize the path complexities found from the first model. These were combined to a single one using weighted assemblage. The second model was proposed using the ant colony optimization algorithm as multi-objective time-varied shortest path approach using modified Dijkstra algorithm. Studies in (Fu and Luo 2015) merged ACO with Emergency Material Scheduling (EMS) to come up with an emergency rescue system capable of performing various functionalities, including material management, city information management, vehicle management, emergency calls, and rescue. A multi-objective optimization approach was developed and used in solving a multi-colony ACO. It produces and an optimized result of a Pareto solution space from data envelopment analysis (DEA), in a timely manner. One of the disadvantages of ACO is its uncertainty on time of convergence. The approach proposed in (Huang and Yu 2017) is aimed at overcoming this. A new pheromone and greedy heuristic function for lot-splitting of jobs is suggested, coupled with a nimble local search technique for the improvements of solution quality. A particle swarm optimization (PSO)-based algorithm for adaptive tuning of parameters is also proposed here.

\subsubsection{PARTICLE SWARM OPTIMIZATION}

Particle swarm optimization is based on population-level characteristics of communal animals, originally proposed by Kennedy and Eberhart(Kennedy, Eberhart, and Gov 1995). It simulates the spatial behavior of animals living in groups, such as a flock of birds or a school of fish. In this setting, individuals in the group move toward a common target without a pre-defined or agreed controlling agent. Individuals in the group (called swarm) are called particles. Each particle represents one approach to providing a comprehensive solution to the group's problem. Studies in (Ai and Kachitvichyanukul 2009)and(Marinakis, Iordanidou, and Marinaki 2013) propose two approaches, for the application of PSO to emergency vehicle routing problems and settings. They involved consideration of stochastic demands from affected locations. After initial routing design with PSO, vehicles leaving the depot are expected to load more than the route requirement. The vehicles may proceed to satisfy the demand of locations or decide to return to the depot for re-loading. This decision is made at each stop, when the demands from unvisited locations are checked. Eight different topological designs and equations are used.

Generally, relief routing, with associated logistics is one of the most vital operations in emergency management. Bozorgi-Amiri et al (Bozorgi-Amiri et al. 2013) discusses the relief chain design problem, with consideration of four uncertain parameters: demands, supplies, procurement cost, and transportation cost. It also takes uncertainties in demand locations and facility security into consideration. A new comprehensive and robust optimization model to tackle the disaster relief logistics problem is introduced as a mixed-integer, nonlinear programming (MINLP) problem. It aims to minimize not only the combined costs of location, procurement, transportation, holding, and shortage but also the variance of the combined cost. To solve the model, a particle swarm optimization approach is proposed, where the position of one particle is considered one solution; each particle is regarded as an individual; swarm comprises of particles. It was shown that the proposed model is useful using the results obtained.

\subsubsection{FIREFLY ALGORITHM}

The firefly algorithm (FA) is a swarm intelligent and metaheuristic algorithm, inspired by the characteristic behaviors of fireflies. It was initially developed by Yang in 2008 and has been successfully applied to many 
continuous optimization problems. Operations of the FA are based on few principles: That Fireflies are unisexual and are attracted to other fireflies regardless of their sex. Brighter fireflies tend to attract less brighter, as fireflies' attractiveness is directly proportional to the brightness and decreases with distance. This ensures that all the fireflies move toward one with higher luminescence until the population converges to brightest one. If there is no brighter firefly, the movement is assumed to be random. The objective function of FA involves three parameters of attractiveness, randomization, and absorption. Attractiveness parameter is based on light intensity between two fireflies and defined with exponential functions. The objective function of a firefly $i$, which is attracted to another brighter firefly $j$, according to studies in (Yang and Karamanoglu 2013), is determined by

$$
x_{i}^{t+1}=x_{i}^{t}+\beta_{0} e^{-y r_{i j}^{2}}\left(x_{j}^{t}-x_{i}^{t}\right)+\alpha x \varepsilon_{i}^{t}
$$

Where $\beta_{0} e^{-y r_{i j}^{2}}$ is the relative luminance value. $\beta_{0}$ is the maximum attraction or attractive ness when distance $r=0$ and is generally taken as $\beta_{0}=1 . y$ is the light absorption coefficient; $y \in[0.01,100]$. It determines the convergence speed and optimization effect of the algorithm.

Some approaches have been proposed, based on amended firefly algorithm. Gharib et al (Gharib et al. 2018) proposed a three-stage approach to solving relief distribution problem in damaged infrastructure situation. Two multi-objective meta-heuristic approaches were developed for implementing quick distribution of vital commodities. The algorithms are the Non-dominated Sorting Genetic Algorithm (NSGA-II), the Multi-Objective Firefly Algorithm (MOFA). A performance comparison of the two algorithms found that the MOFA converges faster to a solution and posts higher accuracy than the NSGA-II. An evolutionary discrete version of the Firefly Algorithm (EDFA) is presented in (Osaba, Carballedo, and Yang 2016) for solving emergency Vehicle Routing Problem with Time Windows (VRPTW). The technique incorporates some novel route optimization operators, capable of implementing arc exchange and route minimization. The route reduction helps in effectively managing emergency deliveries in post-disaster relief operation. Performance comparison with 56 instances of VRPTW shows the effectiveness of the proposed algorithm. An essential part of the logistic distribution in a post-disaster emergency is the timely delivery of relief aid to the people in need. A firefly algorithm-based methodology is proposed in (Pan et al. 2013) and includes the coding and design of the disturbance mechanism of elicit fireflies. The technique was applied in solving a VRPTW, with suggestions for its applications in emergency situations. A similar application of the firefly algorithm in solving the Traveling Salesman Problem is presented in (Jaradat, Matalkeh, and Diabat 2019). Modifications of the initial FA, in terms of distance function, and movement of the Fireflies are done in the study. It was used in solving the Travelling Salesman Problem and could be adapted to routing post-disaster relief aid distribution. The modified algorithm achieves an improved solution in contrast to the initial solution where the fireflies are distributed over the search space. The authors use Hamming's distance, instead of the Cartesian distance function. The firefly is represented in the form of permutation matrices for relief transportation. It reaches an optimal solution, although local, faster than the original algorithm. A more recent approach to routing of relief distribution is presented in (Jaradat, Matalkeh, and Diabat 2019). It proposes a solution to TSP using the Firefly Algorithm and a clustering technique. The approach involves three major steps of clustering the nodes, finding the optimal path in each cluster, and reconnecting the clusters. It yields a relatively better result.

\subsection{ARTIFICIAL IMMUNE SYSTEMS}

Artificial Immune System (AIS) is a collection of computationally intelligent, rule-based machine learning algorithms. These algorithms are inspired by the principles and processes of the immune system of some living creatures. They have been used in stimulating and unraveling the various complexities of optimization problems. Most research activities on Artificial Immune systems are centered on four variations:- negative selection algorithms; artificial immune networks; clonal selection algorithms; and Danger Theory algorithms. An AIS-based immune multi-affinity network model for solving emergency logistics problem is presented in (Hu 2010). It develops an underling architecture of emergency logistics network for managing the complex collaborative relationship among various emergency logistics components. It aims to maximize time efficiency in delivery, while reducing the cost. Studies in (Knott 1988) extended the traditional AIS algorithm for dual aircrafts path planning, applicable to any large scale disaster and emergencies. The problem considers path finding for aircrafts from different sites, and 
Optimization Models for Emergency Response and Post-Disaster Delivery Logistics: A Review of Current Approaches

ensuring they all arrive at the destination the same time. The approach defines an affinity function for evaluating the threats to different flight path, using crossover operators for diverse search, and selecting the safest. A more recent application of AIS to evacuation in disaster management is presented in (Khalid and Yusof 2015). The approach is for optimizing crowd emergency evacuation in large- scale emergencies. It considers complexities of the disasters situation and helps in solving emergency route planning problem. More research activities are therefore needed to ascertain the effectiveness of the application of AIS algorithms to emergency routing and logistics distribution during emergencies.

\section{OTHER METAHEURISTIC APPROACHES}

Some general metaheuristic approaches have been suggested for VRP variants that have frequent applications to emergencies. Multi-Depot Vehicle Routing Problem (MDVRP) is one of these variants that is regularly used in public health emergencies. A review of the current status of MDVRP is presented by Montoya Torres et al (MontoyaTorres et al. 2015). Studies in (Alonso, Alvarez, and Beasley 2008) presents a site-dependent multi-trip metaheuristic algorithm, with tabu search. The algorithm considers some constraints on accessibility of the selected vehicle to nodes and possibility of the vehicle covering more than one route. A hybrid algorithm for MDVRP with capacity constraint was initially proposed by Contardo et al in (Contardo and Martinelli 2014) and modified by studies in(Stodola 2018). Many hybrid heuristic algorithms, however, have been developed to compute feasible solutions to the VRP, with no consideration for the quality of the solution or route improvement (Cordeau et al, Kumar and Panneerselvam, Belhaiza et al., and Escobar et al.(Cordeau and Laporte 2004; Kumar and Panneerselvam 2012; Belhaiza, M'Hallah, and Brahim 2017; Escobar et al. 2014)). A metaheuristic algorithm, based on both simulated annealing and the genetic approach, was proposed by Seidgar et al in (Seidgar et al. 2018). Some approaches combine bio-inspired algorithms with other heuristic techniques, such as studies by Yao et al(Yao et al. 2017), who applied the hybrid ACO algorithm to a seafood product delivery routing problem. A mixed-integer linear programming model, incorporating a hybridization of simulated annealing and particle swarm optimization algorithms, for allocating and scheduling rescue units in relief operations for a natural disaster is presented in (Sina, Asadi-Gangraj, and Emami 2018).

\section{EMERGING CHALLENGES AND RESEARCH OPPORTUNITIES}

From this survey of recent research activities on the use of approximate optimization models routing of emergency deliveries, it can be found that bio-inspired and intelligent algorithms seem to provide faster and competitive alternatives to conventional algorithms for emergency vehicle routing problem. Evolutionary and genetically based algorithms are also capable of finding high-quality solutions for some set of problems. Some hybrid genetic algorithms, with other metaheuristics approaches, have been proposed and have proven to be effective in obtaining a solution, using available theoretical VRP instances. They are, however, more general in approach, and require relatively higher computational resources and planning. More real-world applications of these approaches are needed to further determine their level of reliability. There are very few real-world benchmark instances publicly available. Artificial immune systems and bio-inspired algorithms represent another promising area of research in emergency and disaster logistics management. These algorithms can be applied to the various areas of emergency planning, including maintenance of communication systems in fast-changing disaster situations. Very little research on the use of AIS and artificial intelligence-based algorithms have been conducted in the context of disaster planning and execution. Further work on this genre or its hybridization will be helpful in the dynamic routing of relief deliveries, mostly in uncertain and unreliable settings, as obtainable in LMICs.

Facility location-allocation problem during disasters is another area that has attracted considerable interest in literature. However, in most papers reviewed, it was considered as a pre-disaster planning activity only. In most developing countries, there is a greater level of reliance on international donors, whenever disasters strike or there is an outbreak of epidemics. These organizations, on arrival with the relief materials, are confronted with the issue of depot allocation-allocation for effectively delivering these relief materials to the affected population. Setting up temporary distribution centers is oftentimes needed. Incorporating depot location as part of post-disaster emergency planning will invariably improve operational efficiency and timeliness. For dynamic routing and handling of uncertainties in disaster situations, considering depot allocation as part of the post-disaster research will be most 
helpful. Moreover, further research activities on the development of comprehensive models for emergency management in uncertain and unreliable conditions, incorporating both pre- and post-disasters planning activities are needed. These models are vital in an under-resource setting where separate and different levels of planning may not be feasible, and sources of relief materials are not certain. Moreover, having many organizations and individuals participating in the relief distribution and emergency management makes the coordination a challenging task. Some agencies, volunteers, and relief transporters may join and leave the relief network dynamically. Robust real-time optimization models that include these uncertainties, could be developed.

Additionally, interventions aimed at mitigating the effects of a disaster and emergency response activities in Low and-Middle Income Countries (LMICs) are usually very daunting and more expensive. According to recent research by an NIH Fogarty-led group, what works in High-Income Countries (HICs) most often do not work in LMICs. More in-depth research activities are required to effectively mitigate losses and efficiently manage frequentlyoccurring public health emergencies in these regions (Mowafi et al. 2019). Optimizing routing of deliveries, as part of the Emergency Care System (ECS) improvement program could greatly reduce morbidity and mortality rate during disasters in LMICs. However, detailed data on infrastructure, road network, available vehicles, and their capacities are largely absent in LMICs. Policymakers and emergency response planners have little evidence-based information to produce a robust, time-sensitive planner. There is, therefore, a dire need for comprehensive research that will produce a reference data repository, and a sample logistics delivery routing plan, using data sets that are peculiar to the region.

\subsection{CONCLUSION}

This paper presents a review of recent optimization models for disaster and emergency logistics. These include techniques for facilities or depot location-allocation models, routes construction, and routes improvement, with emphasis on public health emergencies in under-resourced countries. Optimization models for each of these three vital areas of disaster planning and execution are surveyed, including evolutionary algorithms, bio-inspired or intelligent algorithms, and artificial immune systems. Some suggested amendments, updates, and hybridization of these algorithms were presented. It should be noted that the surveyed papers are representative of key groupings of algorithms that have direct applications in disaster situations of uncertainties as obtainable in LMICS. This limitation may affect some inferences on state-of-the-art research situations. The research gaps are identified, and suggestions for potential future research concentrations are suggested. Also, more real-world applications of these suggested approaches are required to further demonstrate their applicability to uncertain emergency situations.

\section{SOURCES OF FUNDING}

None.

\section{CONFLICT OF INTEREST}

None.

\section{ACKNOWLEDGMENT}

None.

\section{REFERENCES}

[1] AbdAllah, A.M; Essam, D. L; Sarker, R.A. 2017. “On Solving Periodic Re-Optimization Dynamic Vehicle Routing Problems.” Applied Soft Computing 55: 1-12.

[2] Ai, The Jin, and Voratas Kachitvichyanukul. 2009. "Particle Swarm Optimization and Two Solution Representations for Solving the Capacitated Vehicle Routing Problem." Computers and Industrial Engineering 56 (1): 380-87. https://doi.org/10.1016/j.cie.2008.06.012. 
Optimization Models for Emergency Response and Post-Disaster Delivery Logistics: A Review of Current Approaches

[3] Al-dahash, Hajer, Menaha Thayaparan, and Udayangani Kulatunga. 2016. "Understanding the Terminologies : Disaster ,." Proceedings of the 32nd Annual ARCOM Conference 2 (September): 1239-48.

[4] Alenezy, Eiman J. 2020. "Solving Capacitated Facility Location Problem Using Lagrangian Decomposition and Volume Algorithm." Advances in Operations Research 2020. https://doi.org/10.1155/2020/5239176.

[5] Alonso, F., M. J. Alvarez, and J. E. Beasley. 2008. "A Tabu Search Algorithm for the Periodic Vehicle Routing Problem with Multiple Vehicle Trips and Accessibility Restrictions." Journal of the Operational Research Society 59 (7): 963-76. https://doi.org/10.1057/palgrave.jors.2602405.

[6] Anisya, A., and Ganda Yoga Swara. 2017. "Implementation of Haversine Formula and Best First Search Method in Searching of Tsunami Evacuation Route." IOP Conference Series: Earth and Environmental Science 97 (1). https://doi.org/10.1088/1755-1315/97/1/012004.

[7] Bagherinejad, Jafar, and Azar Niknam. 2018. "Solving the Competitive Facility Location Problem Considering the Reactions of Competitor with a Hybrid Algorithm Including Tabu Search and Exact Method." Journal of Industrial Engineering International 14 (1): 171-83. https://doi.org/10.1007/s40092-017-0216-8.

[8] Balcik, B., and B. M. Beamon. 2008. "Facility Location in Humanitarian Relief." International Journal of Logistics Research and Applications 11 (2): 101-21. https://doi.org/10.1080/13675560701561789.

[9] Barzinpour, Farnaz, Mohsen Saffarian, Ahmad Makoui, and Ebrahim Teimoury. 2014. "Metaheuristic Algorithm for Solving Biobjective Possibility Planning Model of Location-Allocation in Disaster Relief Logistics." Journal of Applied Mathematics 2014. https://doi.org/10.1155/2014/239868.

[10] Belhaiza, Slim, Rym M'Hallah, and Ghassen Ben Brahim. 2017. "A New Hybrid Genetic Variable Neighborhood Search Heuristic for the Vehicle Routing Problem with Multiple Time Windows." 2017 IEEE Congress on Evolutionary Computation, CEC 2017 - Proceedings, 1319-26. https://doi.org/10.1109/CEC.2017.7969457.

[11] Ben-Akiva, Moshe E., Song Gao, Zheng Wei, and Yang Wen. 2012. "A Dynamic Traffic Assignment Model for Highly Congested Urban Networks." Transportation Research Part C: Emerging Technologies 24: 62-82. https://doi.org/10.1016/j.trc.2012.02.006.

[12] Boloori Arabani, Alireza, and Reza Zanjirani Farahani. 2013. "Facility Location Dynamics: An Overview of Classifications and Applications." Computers and Industrial Engineering 62 (1): 408-20. https://doi.org/10.1016/j.cie.2011.09.018.

[13] Bonabeau, E; Dorigo, M; Theraulaz, G. 2000. "Inspiration for Optimization from Social Insect Behaviour." Nature 406 (6791): 38-43.

[14] Boonmee, Chawis, Mikiharu Arimura, and Takumi Asada. 2017. "Facility Location Optimization Model for Emergency Humanitarian Logistics.” International Journal of Disaster Risk Reduction 24 (September): 48598. https://doi.org/10.1016/J.IJDRR.2017.01.017.

[15] Bozorgi-Amiri, Ali, M.S Jabal-Ameli, Mahdi Heydari, and Mehdi Alinaghian. 2013. "A Modified Particle Swarm Optimization for Disaster Relief Logistics under Uncertain Environment.” International Journal of Advanced Manufacturing Technology 60: 357-371.

[16] Campbell, Ann Melissa, Dieter Vandenbussche, and William Hermann. 2010. "Routing for Relief Efforts." Transportation Science 42 (2): 127-45. https://doi.org/10.1287/trsc.1070.0209.

[17] Caunhye, Aakil M., Xiaofeng Nie, and Shaligram Pokharel. 2012. "Optimization Models in Emergency Logistics: A Literature Review.” Socio-Economic Planning Sciences $46 \quad$ (1): 4-13. https://doi.org/10.1016/j.seps.2011.04.004.

[18] Center for Disease Control and Prevention (CDC): Emergency Preparedness Report. 2016. "Receiving, Distributing, and Dispensing Strategic National Stockpile Assets." Washington DC. https://www.orau.gov/sns/v11/ReceivingDistributingDispensingSNSAssets_V11.pdf.

[19] Chen, Huey Kuo, Che Fu Hsueh, and Mei Shiang Chang. 2006. "The Real-Time Time-Dependent Vehicle Routing Problem.” Transportation Research Part E: Logistics and Transportation Review 42 (5): 383-408. https://doi.org/10.1016/j.tre.2005.01.003.

[20] Church, R. Velle, C. R. 1974. “The Maximum Covering Location Problem.” Pap. Sci 32: 101-18.

[21] Contardo, Claudio, and Rafael Martinelli. 2014. "A New Exact Algorithm for the Multi-Depot Vehicle Routing Problem under Capacity and Route Length Constraints." Discrete Optimization 12 (May): 129-46. https://doi.org/10.1016/J.DISOPT.2014.03.001.

[22] Cordeau, Jean-Franc ßois, and Gilbert Laporte. 2004. "A Tabu Search Heuristic for the Static Multi-Vehicle Diala-Ride Problem." Transportation Research Part B 37 37: 579-94. https://doi.org/10.1016/S01912615(02)00045-0. 
Sampson E. Akwafuo, Armin R. Mikler, and Fariba A. Irany

[23] Dantzig, G B, and J H Ramser. 1959. “The Truck Dispatching Problem.” Management Science Series 6 (1): 80 91. https://andresjaquep.files.wordpress.com/2008/10/2627477-clasico-dantzig.pdf.

[24] Escobar, John Willmer, Rodrigo Linfati, Paolo Toth, and Maria G. Baldoquin. 2014. "A Hybrid Granular Tabu Search Algorithm for the Multi-Depot Vehicle Routing Problem." Journal of Heuristics 20 (5): 483-509. https://doi.org/10.1007/s10732-014-9247-0.

[25] Ferrer, José M., M. Teresa Ortuño, and Gregorio Tirado. 2020. "A New Ant Colony-Based Methodology for Disaster Relief." Mathematics 8 (4): 1-23. https://doi.org/10.3390/math8040518.

[26] Fu, Z., and D Luo. 2015. "The Logistics Distribution Route Research of Ant Colony Algorithm." Computer Knowledge and Technology 269 (2): 501-15.

[27] Gai, Wen Mei, Zhong An Jiang, Yun Feng Deng, Jing Li, and Yan Du. 2015. “Multiobjective Route Planning Model and Algorithm for Emergency Management." Mathematical Problems in Engineering 2015. https://doi.org/10.1155/2015/565403.

[28] Gao, S; Wang, Y; Cheng, J; Inazumi, Y; Tang, Z. 2017. "Ant Colony Optimization with Clustering for Solving the Dynamic Location Routing Problem.” Pplied Mathematics and Computation 285: 149-73.

[29] Gharib, Z, A Bozorgi-Amiri, R Tavakkoli-Moghaddam, and E Najaa. 2018. "A Cluster-Based Emergency Vehicle Routing Problem in Disaster with Reliability.” Scientia Iranica E 25 (4): 2312-30. https://doi.org/10.24200/sci.2017.4450.

[30] Han, Chuan Feng, and Chao Zhang. 2009. "Genetic Algorithm for Solving Problems in Emergency Management." 5th International Conference on Natural Computation, ICNC 20094 (September 2009): 25964. https://doi.org/10.1109/ICNC.2009.333.

[31] Han, Yunjun, Xiaohong Guan, and Leyuan Shi. 2011. "Optimization Based Method for Supply Location Selection and Routing in Large-Scale Emergency Material Delivery." IEEE Transactions on Automation Science and Engineering 8 (4): 683-93. https://doi.org/10.1109/TASE.2011.2159838.

[32] Hsueh, Che-Fu, Huey-Kuo Chen, and Huey-Wen Chou. 2008. "Dynamic Vehicle Routing for Relief Logistics in Natural Disasters." In Vehicle Routing Problem. InTech. https://doi.org/10.5772/5641.

[33] Hu, Zhi-Hua. 2010. "A Network for Emergency Logistics Management Inspired by Immune Multi-Affinity Model." In 2009 International Conference on Information Management, Innovation Management and Industrial Engineering, 22-25. https://doi.org/10.1109/ICIII.2009.467.

[34] Huang, Rong Hwa, and Tung Han Yu. 2017. "An Effective Ant Colony Optimization Algorithm for MultiObjective Job-Shop Scheduling with Equal-Size Lot-Splitting.” Applied Soft Computing Journal 57: 642-56. https://doi.org/10.1016/j.asoc.2017.04.062.

[35] Hvattum, Lars Magnus, Arne Løkketangen, and Gilbert Laporte. 2007. "A Branch-and-Regret Heuristic for Stochastic and Dynamic Vehicle Routing Problems." Networks 49 (4): 330-40. https://doi.org/10.1002/net.20182.

[36] Jaradat, Ameera;, Bara'ah; Matalkeh, and Waed Diabat. 2019. "Solving Traveling Salesman Problem Using Firefly Algorithm." In 2019 IEEE Jordan International Joint Conference on Electrical Engineering and Information Technology (JEEIT).

[37] Jotshi, Arun, Qiang Gong, and Rajan Batta. 2009. "Dispatching and Routing of Emergency Vehicles in Disaster Mitigation Using Data Fusion." Socio-Economic Planning Sciences $43 \quad$ (1): 1-24. https://doi.org/10.1016/j.seps.2008.02.005.

[38] Keim, Mark E. 2008. "Building Human Resilience. The Role of Public Health Preparedness and Response As an Adaptation to Climate Change." American Journal of Preventive Medicine 35 (5): 508-16. https://doi.org/10.1016/j.amepre.2008.08.022.

[39] Kennedy, James, Russell Eberhart, and Bls Gov. 1995. “Particle Swarm Optimization.” In Proceedings of 1995 IEEE International Conference on Neural Networks, 1942-1948.

[40] Khalid, Mohd Nor Akmal, and Umi Kalsom Yusof. 2015. “An Artificial Immune Approach for Optimizing Crowd Emergency Evacuation Route Planning Problem.” ICAART 2015 - 7th International Conference on Agents and Artificial Intelligence, Proceedings 2 (August): 503-8. https://doi.org/10.5220/0005275305030508.

[41] Knott, R. P. 1988. "Vehicle Scheduling for Emergency Relief Management: A Knowledge-Based Approach." Disasters 12 (4): 285-93. https://doi.org/10.1111/j.1467-7717.1988.tb00678.x.

[42] Kumar, Suresh Nanda, and Ramasamy Panneerselvam. 2012. "A Survey on the Vehicle Routing Problem and Its Variants.” Intelligent Information Management 04 (03): 66-74. https://doi.org/10.4236/iim.2012.43010. 
Optimization Models for Emergency Response and Post-Disaster Delivery Logistics: A Review of Current Approaches

[43] Li, Xia, and Anthony Gar On Yeh. 2006. "Integration of Genetic Algorithms and GIS for Optimal Location Search." International Journal of Geographical Information Science 19 (5): 581-601. https://doi.org/10.1080/13658810500032388.

[44] Li, Ziyao. 2010. "Improved Ant Colony Optimization for Emergency Rescue VRP Based on Matlab." ICCASM 2010 - 2010 International Conference on Computer Application and System Modeling, Proceedings 14 (Iccasm): V14-497-V14-499. https://doi.org/10.1109/ICCASM.2010.5622143.

[45] Lin, Yeh-Hung;, Rajan Batta, and Peter Rogerson. 2012. "A Logistics Model for Emergency Supply of Critical Items in the Aftermath of a Disaster." Socio-Economic Planning Sciences 45 (4): 132-45.

[46] Ma, Yongjie, Tian Ye, and Wenjing Hou. 2011. "Dynamic Path Optimization of Emergency Transport Based on Hierarchical Genetic Algorithm." Advances in Swarm Intelligence, 445-52.

[47] Mangla, Monika;, Rakhi; Akhare, and Smita Sanjay. 2015. "Implementing Genetic Algorithm to Solve Facility Location Problem." Mathematica.

[48] Marinakis, Yannis, Georgia Roumbini Iordanidou, and Magdalene Marinaki. 2013. "Particle Swarm Optimization for the Vehicle Routing Problem with Stochastic Demands." Applied Soft Computing Journal 13 (4): 1693-1704. https://doi.org/10.1016/j.asoc.2013.01.007.

[49] Mete, Huseyin Onur Zelda B. Zabinsky. 2010. "Stochastic Optimization of Medical Supply Location and Distribution in Disaster Management." International Journal of Production Economics 1 (126): 78-84.

[50] Montoya-Torres, Jairo R., Julián López Franco, Santiago Nieto Isaza, Heriberto Felizzola Jiménez, and Nilson Herazo-Padilla. 2015. "A Literature Review on the Vehicle Routing Problem with Multiple Depots." Computers and Industrial Engineering 79: 115-27. https://doi.org/10.1016/j.cie.2014.10.029.

[51] Mowafi, Hani, Christine Ngaruiya, Gerard O’Reilly, Olive Kobusingye, and Vikas Kapil. 2019. “Emergency Care Surveillance and Emergency Care Registries in Low-Income and Middle-Income Countries: Conceptual Challenges and Future Directions for Research Analysis." BMJ Global Health 4: 1442. https://doi.org/10.1136/bmjgh-2019-001442.

[52] ogunwolu, L;Sosimi, VA. 2018. “Optimal Routing for Automated Emergency Vehicle Response for Incident Intervention." Journal of Applied Sciences and Environmental Management 22 (5): 749-54. https://www.ajol.info/index.php/jasem/article/view/172696/162093.

[53] Osaba, Eneko, Roberto Carballedo, and Xin-she Yang. 2016. "An Evolutionary Discrete Firefly Algorithm with Novel Operators for Solving the Vehicle Routing Problem with Time Windows" 637 (February 2018): 1-20. https://doi.org/10.1007/978-3-319-30235-5.

[54] Pan, Fengshan, Chunming Ye, Kefeng Wang, and Jiangbo Cao. 2013. "Research on the Vehicle Routing Problem with Time Windows Using Firefly Algorithm." Journal of Computers (Finland) 8 (9): 2256-61. https://doi.org/10.4304/jcp.8.9.2256-2261.

[55] Pichpibul, Tantikorn, and Ruengsak Kawtummachai. 2013. "A Heuristic Approach Based on Clarke-Wright Algorithm for Open Vehicle Routing Problem." The Scientific World Journal 2013. https://doi.org/10.1155/2013/874349.

[56] Pillac, Victor, Michel Gendreau, Christelle Guéret, Andrés L Medaglia, Christelle Gú, and Andrés L Medaglia. 2013. "A Review of Dynamic Vehicle Routing Problems." European Journal of Operational Research 225 (1): 1-11. https://doi.org/10.1016/j.ejor.2012.08.015ï.

[57] Prasanth, R S S, and Hans Raj Kandikonda. 2015. “Using Bio-Inspired Algorithm” 116 (September): 663-69.

[58] Psaraftis, Harilaos N. 1980. "Dynamic Programming Solution To the Single Vehicle Many-To-Many Immediate Request Dial-a-Ride Problem.” Transportation Science 14 (2): 130-54. https://doi.org/10.1287/trsc.14.2.130.

[59] Radianti, Jaziar, Ole Christoffer Granmo, Parvaneh Sarshar, Morten Goodwin, Julie Dugdale, and Jose J. Gonzalez. 2014. "A Spatio-Temporal Probabilistic Model of Hazard- and Crowd Dynamics for Evacuation Planning in Disasters.” Applied Intelligence 42 (1): 3-23. https://doi.org/10.1007/s10489-014-0583-4.

[60] Rawls, Carmen G., and Mark A. Turnquist. 2010. "Pre-Positioning of Emergency Supplies for Disaster Response." Transportation Research Part B: Methodological 44 (4): 521-34. https://doi.org/10.1016/j.trb.2009.08.003.

[61] Ritchie, H; Roser, Max. 2019. "Natural Disasters - Our World in Data." The B.E. Journal of Economic Analysis \& Policy 8 (1). https://doi.org/10.2202/1935-1682.1903.

[62] Seidgar, Hany, Mehrdad Ezzati, Morteza Kiani, and Reza Tavakkoli-moghaddam. 2018. "An Efficient Genetic Algorithm For Two-Stage Hybrid Flow Shop Scheduling With Preemption And Sequence Dependent Setup International Journal of Engineering Technologies and Management Research 
Time." Journal of Mathematics and Computer Science $06 \quad$ (04): 251-59. https://doi.org/10.22436/jmcs.06.04.01.

[63] Sever, Derya, Lei Zhao, Nico Dellaert, Emrah Demir, Tom Van Woensel, and Ton De Kok. 2018. "The Dynamic Shortest Path Problem with Time-Dependent Stochastic Disruptions." Transportation Research Part C: Emerging Technologies 92 (July): 42-57. https://doi.org/10.1016/j.trc.2018.04.018.

[64] Shen, Ling, Fengming Tao, Yuhe Shi, and Ruiru Qin. 2019. "Optimization of Location-routing Problem in Emergency Logistics Considering Carbon Emissions." International Journal of Environmental Research and Public Health 16 (16). https://doi.org/10.3390/ijerph16162982.

[65] Shen, Maged;, Fernando; Dessouky, and JiaZhihong Ordóñez. 2007. "Rapid Distribution of Medical Supplies." International Series in Operations Research \& Management Science, 307-38.

[66] Simić, Dragan, and Svetlana Simić. 2012. "Hybrid Artificial Intelligence Approaches on Vehicle Routing Problem in Logistics Distribution." Lecture Notes in Computer Science (Including Subseries Lecture Notes in Artificial Intelligence and Lecture Notes in Bioinformatics) 7208 LNAI (PART 1): 208-20. https://doi.org/10.1007/978-3-642-28942-2_19.

[67] Sina, Nayeri;, Ebrahim; Asadi-Gangraj, and S Emami. 2018. "Metaheuristic Algorithms to Allocate and Schedule of the Rescue Units in the Natural Disaster with Fatigue Effect." Neural Computing and Applications 31 (10): 1-21. https://doi.org/10.1007/s00521-018-3599-6.

[68] Stodola, Petr. 2018. "Using Metaheuristics on the Multi-Depot Vehicle Routing Problem with Modified Optimization Criterion." Algorithms 11 (5): 74. https://doi.org/10.3390/a11050074.

[69] Tragantalerngsak, Suda, John Holt, and Mikael Ronnqvist. 2001. "An Exact Method for the Two-Echelon , Single- Source , Capacitated Facility Location Problem.” European Journal of Operational Research 123: 47389.

[70] Urbanovsky, Joshua Charles. 2018. "Computational Methods to Optimize High-Consequence Variants of the Vehicle Routing Problem for Relief Networks in Humanitarian Logistics." PhD Dissertation, University of North Texas. https://digital.library.unt.edu/ark:/67531/metadc1248473/m2/1/high_res_d/URBANOVSKYDISSERTATION-2018.pdf.

[71] Walia, S S, K U S Somarathna, R Hendricks, A Jackson, and N Nagarur. 2018. "Optimizing the Emergency Delivery of Medical Supplies with Unmanned Aircraft Vehicles," no. May: 1588-93. https://www.scopus.com/inward/record.uri?eid=2-s2.0-

85054014297\&partnerID=40\&md5=9f6044bd6717a0e5c5a00d21f2a1e9a0.

[72] Wang, D., Zhang, G. 2006. "Model and Algorithm to Optimize Location of Catastrophic Rescue Center." Frontiers of Electrical and Electronic Engineering 26 (1): 265-69.

[73] Xiang, Xiaoshu, Jianfeng Qiu, Jianhua Xiao, and Xingyi Zhang. 2020. "Demand Coverage Diversity Based Ant Colony Optimization for Dynamic Vehicle Routing Problems." Engineering Applications of Artificial Intelligence 91 (March): 103582. https://doi.org/10.1016/j.engappai.2020.103582.

[74] Xu, Haitao; Pu Pan; Duan Feng. 2018. "Dynamic Vehicle Routing Problems with Enhanced Ant Colony Optimization." Discrete Dynamics in Nature and Society 2018 (1): 1-13.

[75] Yang, S; Tinos, R. 2007. "A Hybrid Immigrants Scheme for Genetic Algorithms in Dynamic Environments." International Journal of Automation and Computing 4 (3): 243-55.

[76] Yang, Xin-She, and Mehmet Karamanoglu. 2013. "Swarm Intelligence and Bio-Inspired Computation: An Overview." In Swarm Intelligence and Bio-Inspired Computation: Theory and Applications, 3-23. London.

[77] Yao, Baozhen, Qianqian Yan, Mengjie Zhang, and Yunong Yang. 2017. "Improved Artificial Bee Colony Algorithm for Vehicle Routing Problem with Time Windows." PLoS ONE 12 (9): 1-18. https://doi.org/10.1371/journal.pone.0181275.

[78] Yu, B; Yang, Z; Yao, B. 2009. “An Improved Ant Colony Optimization for Vehicle Routing Problem.” European Journal of Operational Research 196 (1): 172-77.

[79] Yuan, Yuan, and Dingwei Wang. 2009. "Path Selection Model and Algorithm for Emergency Logistics Management." Computers and Industrial Engineering 56 (3): 1081-94. https://doi.org/10.1016/j.cie.2008.09.033.

[80] Zheng, Yu Jun, Sheng Yong Chen, and Hai Feng Ling. 2015. "Evolutionary Optimization for Disaster Relief Operations: A Survey." Applied Soft Computing Journal 27: 553-66. https://doi.org/10.1016/j.asoc.2014.09.041. 